\title{
Role of nutraceuticals in health and disease prevention: A review
}

\author{
Krishan Kumar $^{*}$ and Sarvesh Kumar ${ }^{2}$ \\ ${ }^{1}$ Department of Food Technology, Akal College of Agriculture, \\ Eternal University, Baru Sahib, Distt. Sirmour, HP \\ ${ }^{2}$ Department of Food Technology, Baba Farid Institute of Technology, Dehradun-248007
}

*E-mail : krishankumar02007@gmail.com

\begin{abstract}
The term nutraceutical was originally defined by Dr. Stephen L. De Felice, founder and chairman of the Foundation of Innovation Medicine (FIM), Crawford, New Jersey. About 2000 years ago, Hippocrates correctly emphasized "Let food be your medicine and medicine be your food". Nutraceuticals are medicinal foods that play a role in maintaining well being, enhancing health, modulating immunity and thereby preventing as well as treating specific diseases. Thus the field of nutraceutical can be envisioned as one of the missing blocks in the health benefit of an individual. Probiotics are ingested for their purported positive advantages in the digestive tract and/or systemic area like the liver, brain, vagina or blood stream. Antioxidants are very essential in the treatment of almost all diseases because most chronic diseases carry with them a great pact of oxidative stress. Oxidative stress is accelerated by the ageing process along with lack of dietary antioxidants. Flavonoids have anti-cancerous properties by acting as antioxidants. Phytochemicals have specific actions and can be used variously for e.g. as antioxidants and have a positive effect on health. Recently, much attention has been given to phytochemicals that possess cancer preventive properties. Omega-3 polyunsaturated fatty acids (PUFAs) have been shown to decrease the production of inflammatory eicosanoids, cytokines and reactive oxygen species, possess immunomodulatory effects and attenuate inflammatory diseases. With the evolution of "Smart Nutraceuticals", a Futuristic "Physician's Desk Reference" would contain information on individual genetic profiles to be matched with specific nutritional interventions as well. This would be a vast improvement over current nutritional recommendations which being too generalized are reported to benefit only $60 \%$ of population.
\end{abstract}

Key Words: Nutraceuticals, medicine, health, antioxidants, phytochemicals, diseases

Paper cited: Kumar, Krishan and Kumar, Sarvesh (2015). Role of Nutraceuticals in Health and disease prevention: A review. South Asian J. Food Technol. Environ. 1(2):116-121.

The term nutraceutical was originally defined by Dr. Stephen L. De Felice, founder and chairman of the Foundation of Innovation Medicine (FIM), Crawford, New Jersey. Since the term was coined by Dr. De Felice, its meaning has been modified by Health Canada which defines nutraceutical as: a product isolated or purified from foods, and generally sold in medicinal forms not usually associated with food and demonstrated to have a physiological benefit or provide protection against chronic disease. Examples are $\beta$-carotene and lycopene. Dr Stephen DeFelice coined the term "Nutraceutical" from "Nutrition" and "Pharmaceutical" in 1989. The term nutraceutical is being commonly used in marketing but has no regulatory definition (Gupta et al., 2013).

About 2000 years ago, Hippocrates correctly emphasized "Let food be your medicine and medicine be your food". In the past five years, the world has witnessed the explosive growth of a multibillion dollar industry known as nutraceutical. The term "nutraceutical" combines the word "nutrient" (a nourishing food or food component) with "pharmaceutical" (a medical drug).
Nutraceuticals have received considerable interest in recent times because of their presumed safety and potential nutritional and pharmaceutical value. Nutraceuticals are substances which are not traditionally recognized nutrients but which have positive physiological effects on the human body. They are claimed to possess multiple therapeutic benefits. The medicinal plants represent one of the important fields of traditional medicine all over the world and hence an established constituents of nutraceuticals (Singh and Geetanjali, 2013).

The interface between the nutritional environment and cellular/ genetic processes is termed as 'nutrigenomics'. It provides a molecular enlightenment of phytochemicals benefitting human health by altering the expression or constitution of genes. This leads to alteration in initiation, development and progression of different diseases. Thus, nutrigenomics is very important in the role of nutraceuticals against ageing and different diseases by proving genetic information (Dahiya, 2013).

Foods and nutrients play a vital role in normal functioning of the body. They are helpful in maintaining the health of the individual and in 
reducing the risk of various diseases. Nutraceuticals are medicinal foods that play a role in maintaining well being, enhancing health, modulating immunity and thereby preventing as well as treating specific diseases. Thus the field of nutraceutical can be envisioned as one of the missing blocks in the health benefit of an individual. It has been scientifically proved and supported by various research articles that nutraceutical are efficacious to treat and prevent various disease conditions (Rama et al., 2006).

\section{Classification of Nutraceuticals}

Nutraceuticals are non-specific biological therapies used to promote wellness, prevent malignant processes and control symptoms. They are classified as follows:

\section{(a) Nutrients}

Nutrients are substances such as vitamins, minerals, amino acids and fatty acids with established nutritional functions. Most vegetables, whole grain cereals, dairy products, fruits and animal products such as meat, poultry, contain vitamins and are helpful in curing heart diseases, stroke, cataracts, osteoporosis, diabetes and cancer. Minerals found in plant, animal and dairy products are useful in osteoporosis, anaemia and build strong bones, teeth, muscles, improve nerve impulses and heart rhythm. Flax seed and salmon contain fatty acids omega-3 PUFAs, and are potent controllers of the inflammatory processes, maintenance of brain function and reduce cholesterol deposition (Singh and Sinha, 2012). The most commonly known nutrients are antioxidant, water and fat-soluble vitamins. Many potential benefits have been attributed to antioxidant use in the form of dietary intake or supplementation. Antioxidants, in general, may be useful in the prevention of cancer and cerebrovascular disease. High dietary intake of vitamin E may prevent Parkinson's disease (Sapkale et al., 2012).

\section{(b) Herbals}

Herbals are as old as human civilization and they have provided a complete storehouse of remedies to cure acute and chronic diseases. The knowledge of herbals has accumulated over thousands of years so that today we possess many effective means of ensuring health care (Stauffer, 1999). Nutraceuticals holds a great promise to improve health and prevent chronic diseases with the help of herbals. Some examples are willow bark (Salix nigra), having active component as salicin, which is anti-inflammatory, analgesic, antipyretic, astringent and antiarthritic. Parsley (Petroselinum cripsum) contains flavnoids (apiol, psoralen) and is diuretic, carminative and anti-pyretic. Peppermint (Mentha piperita) contains menthol as an active component and cures cold and flu. Lavender (Lavandula angustifolia) contains tannin which is helpful in curing depression, hypertension, stress, cold, cough and asthma. Cranberries (Vaccinium erythrocarpum) contain proanthocyanadin and are found to be useful in cancer, ulcers and urinary tract infections (Singh and Sinha, 2012).

(c) Dietary Supplements

Dietary supplements are products administered through mouth that contain a dietary ingredient intended to add something to the foods you eat. As defined by the Dietary Supplement Health and Education Act (DSHEA, 1994), "a product that contains one or more of the dietary ingredients such as vitamin, mineral, herb, or other botanical, and amino acid (protein) also includes any possible component of the diet as well as concentrates, constituents, extracts or metabolites of these compounds." (Sapkale et al., 2012). Examples of dietary supplements are black cohosh for menopausal symptoms, ginkgo biloba for memory loss and glucosamine/chondroitin for arthritis. They also serve specific functions such as sports nutrition, weight-loss supplements and meal replacements. Supplement ingredients may contain vitamins, minerals, herbs or other botanicals, amino acids, enzymes, organ tissues, gland extracts, or other dietary substances. They are available in different dosage forms, including tablets, capsules, liquids, powders, extracts, and concentrates (Chintale et al., 2013)

\section{Role of bacterial nutraceuticals}

Bacteria such as lactic acid bacteria (LAB) are used all over the world in a large variety of industrial food fermentations (Chelule et al., 2010). Some of the starter bacteria used in dairy fermentation are the yoghurt bacterium Streptococcus thermophilus and the cheese and butter (milk) bacterium Lactococcus lactis. This property offers the possibility to fortify fermented dairy products with folate by natural means i.e. without the addition of food supplements. Some lactic acid bacteria, such as Lactococcus lactis, are able to produce and excrete riboflavin into the surrounding medium (Sharma and Kumari, 2012)

Probiotics are live microbial food ingredients, which are beneficial to health. They are friendly bacteria that promote healthy digestion and absorption of some nutrients. They act to crowd out pathogens, such as yeasts, other bacteria and viruses that may otherwise cause disease and develop a mutually advantageous symbiosis with the human gastrointestinal tract. They have an antimicrobial effect through modifying the microflora, preventing adhesion of pathogens to the intestinal epithelium, competing for nutrients necessary for pathogen survival, producing an antitoxin effect and reversing some of the consequences of infection on the intestinal epithelium (Singh and Sinha, 2012). An international expert group of the International Life Sciences Institute (ILSI) has evaluated the categorized and published evidence of functionality 
of different probiotics in four areas of (human) application, namely, (i) metabolism, (ii) chronic intestinal inflammatory and functional disorders, (iii) infections, and (iv) allergy (Cencic and Chingwaru, 2010) .

The various types of bacteria that are having the probiotics characteristics are:Lactobacilli, Lactobacillus rhamnosus, Lactobacillus reuteri, Lactobacillus case, Bifidobacterium, Bifidobacterium lactis, Bifidobacterium longum, Bifidobacterium breve, Bifidobacterium infantis, Streptococcus, Lactococcus, Lactococcus platinum, Lactococcus reuteri, Lactococcus agilis, Enterococcus, Saccharomyces, Bacillus, Pediococcus ( Singh et al., 2012). There are large numbers of benefits of using probiotics as nutraceuticals. Some of these benefits include: enhancing bowel function, prevention of colon cancer, lowering cholesterol, lowering of blood pressure, improving immune function, reducing infections, reducing inflammation, improving mineral absorption, preventing growth of harmful bacteria, fighting off diseases like candida and eczema, and many more. As these "friendly bacteria" are beneficial for humans similarly there are large numbers of soil bacteria which act as probiotics for plants. They are helpful in promoting the growth, health and yield of crops (Pandey et al., 2011)

\section{Antioxidants in disease prevention}

Antioxidants are known to defuse free radicals leading to limited risk of Oxidative Stress (OS) and associated disorders. At cellular and molecular levels they inactivate Reactive Oxygen Species (ROS) and under specific low concentration inhibit or delay oxidative processes by interrupting the radical chain reaction of lipid peroxidation. Carotenoids, tocopherols, ascorbates, lipoic acids and polyphenols are strong natural antioxidants with free radical scavenging activity. Endogenous antioxidant enzymes like super oxide dismutase (SOD), catalase, glutathione peroxidase, glutathione reductase, minerals like $\mathrm{Se}, \mathrm{Mn}, \mathrm{Cu}$, $\mathrm{Zn}$, vitamins $\mathrm{A}, \mathrm{C}$ and $\mathrm{E}$, carotenoids, limonoids and polyphenols exert synergistic actions in scavenging free radicals (Prakash and Gupta, 2009).

During the last few years researches has confirmed that many common disease (CVS, diabetes, cataracts, high blood pressure, infertility, respiratory infection and rheumatoid arthritis) are associated with tissue deficiency and/or low dietary levels of compounds called antioxidants which make them an essential part of the nutraceutical market. During oxidation free radicals are generated, these free radicals at a molecular level burn everything they touch. Antioxidants are quite large in number and diverse in nature which opposes the process of oxidation largely by neutralizing free radicals at relatively small concentrations and have the potential to inhibit the oxidants chain reactions and ultimately reconstitute the damaged membranes. Dietary antioxidants and some accessory molecules, such as zinc and certain vitamins are important in maintaining free radical scavenging systems, biosynthetic capacity, membranes, enzymes and DNA. Antioxidants are found in the vegetable oils e.g., Soybean oil, canola oil, corn oil, oat oil, wheat germ oil, palm oil, evening prime rose oil ( Singh et al., 2012).

Antioxidants are very essential in the treatment of almost all diseases because most chronic diseases carry with them a great pact of oxidative stress. Oxidative stress plays a chief job in neurodegenerative diseases such as Alzheimer's disease (AD), Parkinson's disease (PD), and Huntington's disease (HD). Oxidative stress is accelerated by the ageing process along with lack of dietary antioxidants. A huge number of studies have found an association between high dietary antioxidant intake and a decreased risk of $\mathrm{AD}$ which is very imperative because preventing a disease is significantly easier than treating it. Researches suggest that preventing AD is actually not that complex. Treatment with antioxidants is a hopeful loom for slowing disease progression (Klatte et al., 2003).

\section{Phytochemicals as potential nutraceuticals}

Phytochemicals (bioactive non-nutrient plant compounds), have raised interest in human nutrition because of their potential effects as antioxidants, antiestrogenics, anti-inflammatory, immunomodulatory, and anticarcinogenics (Laparra and Sanz, 2010). Majority of foods, such as whole grains, beans, fruits, vegetables and herbs contain phytonutrients/ phytochemicals. These phytochemicals, either alone and/or in combination, have tremendous therapeutic potential in curing various ailments. Phytochemicals with nutraceutical properties present in food are of enormous significance due to their beneficial effects on human health since they offer protection against numerous diseases or disorders such as cancers, coronary heart disease, diabetes, high blood pressure, inflammation, microbial, viral and parasitic infections, psychotic diseases, spasmodic conditions, ulcers, osteoporosis and associated disordersn (Prakash et al., 2012).

They are naturally occurring biochemicals that give plants their color, flavor, smell, and texture, which may help prevent diseases. They are biologically active natural products such as glucosinolates in cruciferous vegetables, limonoids in citrus fruits, lignans in flaxseed, lycopene in tomatoes, and catechins in tea. They all have specific actions and can be used variously for e.g. as antioxidants and have a positive effect on health. Recently, much attention has been given to phytochemicals that possess cancer preventive 
properties (Shukla and Singh, 2007). Besides chemopreventive components in vegetables and fruits, some phytochemicals derived from herbs and spices also have potential anticarcinogenic and antimutagenic activities, among other beneficial health effect. A broad range of phytopharmaceuticals with a claimed hormonal activity, called "phyto-estrogens", is recommended for prevention of prostate/breast cancer (Inti and Faoro, 2006).

Tea contains catechin derivative, amino acid theanine (shown reduced blood pressure in hypertensive rats). Black and green tea both show equal activity in total plasma antioxidant status after single dose. But some study show green tea to be more effective than black. Tea polyphenols have anti-inflammatory, antithrombotic and anti-platelet properties and effective in lowering risk of developing Coronary Heart Diseases (Chaturvedi et al., 2011)

Flavonoids have anticancerous properties by acting as antioxidants. They are found in citrus fruits, soyfoods which are unique dietary source of isoflavones, green tea rich in epigallocatechin gallate and curcuma longa rich in curcumin. The main soybean isoflavones, genistein, daidzein, biochanin inhibits prostate cancer cell growth. Carotenoids and lycopenes are also important chemicals for human health. Because of the unsaturated nature of lycopene it is considered to be a potent antioxidant and a reactive oxygen species (ROS) quencher. Recently, it was reported that fruits and vegetables containing lycopene exert cancer protective effect via a decrease in oxidative and other damage to DNA in humans. Among the carotenes, beta carotene is the most active as antioxidants. $\beta$-Carotene is the more common form and can be found in yellow, orange, and green leafy fruits and vegetables (Pandey et al., 2011).

Flavonoids block the angiotensinconverting enzyme (ACE) that raises blood pressure by blocking the "suicide" enzyme cyclooxygenase that breaks down prostaglandins, they prevent platelet stickiness and hence platelet aggregation. Flavonoids also protect the vascular system and strengthen the tiny capillaries that carry oxygen and essential nutrients to all cells. Flavonoids block the enzymes that produce estrogen, thus reducing the risk of estrogen-induced cancers (Rajasekaran et al., 2008).

In recent years, there is growing evidence that plant-foods polyphenols, due to their biological properties, may be unique nutraceuticals and supplementary treatments for various aspects of type 2 diabetes mellitus. potential efficacies of polyphenols, including phenolic acids, flavonoids, stilbenes, lignans and polymeric lignans, on metabolic disorders and complications induced by diabetes have been discovered (Bahadoran et al., 2013).

\section{Polyunsaturated fatty acids as nutraceuticals}

The use of omega-3 oils/long-chain $\omega 3$ PUFA constitutes one of the most promising developments in human nutrition and disease risk reduction in the past three decades. Long-chain $\omega 3$ PUFA are of great interest because of their effectiveness in prevention and treatment of coronary heart disease, hypertension, diabetes, arthritis and other inflammations, autoimmune disorders mental health and neural function as in depression and schizophrenia and cancers and are essential for maintenance and development of normal growth, especially for the brain and retina. (Shahidi, 2012). Polyunsaturated fatty acids (PUFAs) (which include the omega-3 and omega-6 fatty acids) and phytochemicals also play an important role as healthy dietary bioactive compounds. A balanced PUFA composition of food influences diverse aspects of immunity and metabolism. Moreover, interactions between PUFAs and components of the gut microbiota may also influence their biological roles (Cencic and Chingwaru, 2010).

Omega-3 polyunsaturated fatty acids (PUFAs) have been shown to decrease the production of inflammatory eicosanoids, cytokines and reactive oxygen species, possess immunomodulatory effects and attenuate inflammatory diseases (Sohaimy, 2012). Fatty acids of the omega-3 series (n-3 fatty acids) present in fish are well established dietary components affecting plasma lipids and the major cardiovascular disorders, such as arrhythmias (Rajasekaran et al., 2008). The health-promoting effect of fish oil is now known to be due to the omega-fatty acids, especially omega- 3 and omega6. The main omega-3 fatty acids in fish oil are docosahexaenoic (DHA) and eicosapentaenoic acids (EPA). DHA in particular has been shown to be an important structural component of the brain and contributes to improved memory functions. Recently, increased incorporation of DHA into margarines and baby foods has been promoted to enhance brain memory development; a role in reducing the severity of Alzheimer's disease has been suggested for DHA (Sohaimy, 2012).

\section{Nutraceutical Regulations}

Food regulation is aimed at protecting the consumer's health, increasing economic viability harmonizing well-being and engendering fair trade on foods within and between nations. For nutraceutical industries, two challenges are apparent; regulatory uncertainty and credibility of labeling claims. The food sector in India has been governed by multiple laws enacted at different points of time to complement and supplement each other (Singh and Sinha, 2012). 
Regulations differ between countries. Japan was the first country to recognize functional foods as a separate category when in 1991 it introduced the FOSHU (Foods for Specific Health Use) system to evaluate health claims. This system has valuable aspects: it regulates both safety and health and it demands that the food be analyzed for the amount of effective components (Sohaimy, 2012).

The Indian Health and Dietary Supplement Association was created to represent pharmaceutical, nutraceutical, herbal, direct selling and other service oriented industry companies and plans to affiliate with the International Alliance of Dietary Supplement Associations in the near future. The association is planning a scientific conference to bring the industry and government together to share information, experience and perspectives on the use and regulation of dietary supplements. The pressure from the industry and stakeholders for a single regulatory body and an integrated modern food law, the Food Safety and Standards Act, 2006 (FSSA) was enacted by the Government of India (GOI) after extensive consultation and formal legislative procedures (Singh et al., 2012).

\section{The Future of Nutraceuticals}

The expanding nutraceutical market indicates that end users are seeking minimally processed food with extra nutritional benefits and organoleptic value. This development, in turn, is propelling expansion in the nutraceutical markets globally. The emerging nutraceuticals industry seems destined to occupy the landscape in the new millennium. Its tremendous growth has implications for the food, pharmaceutical, healthcare, and agricultural industries Many scientists believe that enzymes represent another exciting frontier in nutraceuticals. "Enzymes have been underemployed. They are going to be a hot area in the future." Fermentation technology using microbes to create new food products also represents potential. Global trends to healthy products cannot be reversed. Companies taking the lead by investing strategically in science, product development, marketing and consumer education will not go unrewarded (Pandey et al., 2010)

Nutraceuticals supplied through oral or transdermal delivery system would provide well targeted health benefits with optimal bioavailability. With the evolution of "Smart Nutraceuticals", a Futuristic "Physician's Desk Reference" would contain information on individual genetic profiles to be matched with specific nutritional interventions as well. This would be a vast improvement over current nutritional recommendations which being too generalized are reported to benefit only $60 \%$ of population (Ball, 2003).

\section{Conclusion}

The nutraceutical industry is growing at a rate far exceeding expansion in the food and pharmaceutical industries. Public health Authorities consider prevention and treatment with nutraceuticals- as a commanding gadget in maintaining health and to perform against nutritionally induced acute and chronic diseases, thereby promoting optimal health, longevity and quality of life. Future demand of nutraceutical depends on consumer perception of the relationship between diet and disease. Although nutraceuticals have significant promise in the promotion of human health and disease prevention, health professional, nutritionists and regulatory toxicologist should strategically work together to plan appropriate regulation to provide the ultimate health and therapeutic benefit to mankind.

It is very imperative that the nutrients found in many foods, fruits and vegetables are responsible for the well documented health benefits. Evidences indicate that the mechanistic actions of natural compounds involve a wide array of biological processes, including activation of antioxidant defenses, signal transduction pathways, cell survival-associated gene expression, cell proliferation and differentiation and preservation of mitochondrial integrity. It appears that these properties play a crucial role in the protection against the pathologies of numerous age-related or chronic diseases.

In order to have scientific knowledge about the nutraceuticals, publics should be educated, and recommended daily doses of these nutraceuticals should be known by each consumer. Nutraceutical Products is collaborative research effort of pharma, food and chemistry. As healthcare industry is growing in India, growth of nutraceuticals is also increasing because people want to treat their disease by improving their health with the help of Fast Moving Healthcare Goods. Now "nutraceutical a day may keep the doctor away" replace the old proverb "an apple a day will keep the doctor away". Consumers are turning massively to food supplements to improve well being where pharmaceuticals fail.

\section{References}

1. Bahadoran, Z., Mirmira, P. and Azizi, F. (2013). Dietary polyphenols as potential nutraceuticals in management of diabetes: a review. J Diabetes Metab Disorders. 12:43.

2. Ball, D. (2003). Foods of the Future May Be Tailored to Fit. Wall Street J. Jan 23, 2003.

3. Cencic, A. and Chingwaru, W. (2010). The Role of Functional Foods, Nutraceuticals, and Food Supplements in Intestinal Health. Nutrients. 2: 611-625.

4. Chaturvedi, S., Sharma, P.K., Garg, V.K. and Bansal, M. (2011). Role of Nutraceuticals in 
Health Promotion. Intern. J Pharm Tech Res. 3(1): 442-448.

5. Chelule, P.K., Mokoena, M.P. and Gqaleni, N. (2010). Advantages of traditional lactic acid bacteria fermentation of food in Africa. Cur Res Technol Edu Topics App Micro Microbial Biotechnol: 1160-1167.

6. Chintale, A.G., Kadam, V.S., Sakhare, R.S., Birajdar, G.O. and Nalwad, D.N. (2013). Role of Nutraceuticals in Various Diseases: A Comprehensive Review. Intern J Res Pharm Chem. 3(2): 290-299.

7. Dahiya, K. (2013). Nutraceuticals and Their Impact on Human Health. J Plant Biochem Physiol. 1(4):1-3.

8. Gupta, S.K., Yadav, S.K. and Patil, S.M. (2013). Nutraceutical-A Bright Scope and Oppourtunity of Indian Healthcare Market. Intern J Res Develop Pharm Life Sci. 2(4): 478-481

9. Inti, M. and Faoro, F. (2006). Grape phytochemicals: a bouquet of old and new nutraceuticals for human health. Medical Нypo. 67: 833-838.

10. Klatte, E.T., Scharre, D.W., Nagaraja, H.N., Davis, R.A. and Beversdorf, D.Q. (2003). Combination therapy of donepezil and vitamin $\mathrm{E}$ in Alzheimer disease. Alzheimer Dis Assoc Disord. 17: 113-116.

11. Laparra, J.M. and Sanz, Y. (2010). Interactions of gut microbiota with functional food components and nutraceuticals. Pharmacol Res. 61(3): 219-225.

12. Pandey, M., Verma, R.K. and Saraf, S.A. (2010). Nutraceuticals: new era of medicine and health. Asian J Pharmaceut Clinic Res. 3(1): 11-15.

13. Pandey, N., Meena, R.P., Rai, S.K. and Rai, S.P. (2011). Medicinal Plants Derived Nutraceuticals: A Re-Emerging Health Aid. Intern J Pharma and Bio Sci. 2(4): 419- 441.

14. Prakash, D. and Gupta, K.R. (2009). The Antioxidant Phytochemicals of Nutraceutical Importance. The Open Nutraceut J. 2: 20-35.
15. Prakash, D., Gupta, C. and Sharma, G. (2012). Importance of Phytochemicals in nutraceuticals. J Chinese Medicine Res Develop. 1(3): 70-78

16. Rajasekaran, A., Sivagnanam, G. and Xavier, R. (2008). Nutraceuticals as therapeutic agents: A Rev Res J. Pharm. Tech. 1(4): 328340.

17. Rama, C.S., Shirode, A.R., Mundada, A.S. and Kadam, V.J. (2006). Nutraceuticals-an emerging era in the treatment and prevention of cardiovascular diseases. Curr Pharm Biotechnol. 7(10):15-23.

18. Sapkale, A.P., Thorat, M.S., Vir, P.R. and Singh, M.C. (2012). Nutraceuticals-Global status and applications: A Review. Intern J Pharmaceut Chem Sci. 1(3): 1166-118.

19. Shahidi, F. (2012). Nutraceuticals, Functional Foods and Dietary Supplements in Health and Disease. J Food Drug Analysis. 20 (1): 226230.

20. Sharma, R. and Kumari, M. (2012). Bacterial Nutraceuticals-A Beneficial Health Supplement. Cibtech J Biotechnol. 1(2-3):1317.

21. Shukla. Y/ and Singh. M/ (2007) Cancer preventive properties of ginger: a brief review. Food ChemToxicol. 45: 683-690.

22. Singh, F., Kumar, M.S. and Mahadevan, N. (2012). Nutraceuticals: Uplift in Health. Intern J Recent Adv Pharmaceut Res. 2(2): 17-28.

23. Singh, J. and Sinha, S. (2012). Classification, Regulatory Acts and Applications of Nutraceuticals for Health. Intern. J. Pharm Biol Sci. 2(1): 177-187.

24. Singh, R. and Geetanjali (2013). Nutraceuticals: Promising Health Product. Intern Res J Med Sci. 1(1): 14-17.

25. Sohaimy, S.A. (2012). Functional Foods and Nutraceuticals-Modern Approach to Food Science. World App Sci J. 20(5): 691-708.

26. Stauffer, J.E. (1999). Nutraceuticals. Cereal Foods World. 44:115-117. 